\title{
Quantized circular motion of a trapped Bose-Einstein condensate: coherent rotation and vortices
}

\author{
Karl-Peter Marzlin and Weiping Zhang \\ School of Mathematics, Physics, Computing and Electronics, Macquarie University, Sydney, NSW 2109,
} Australia

\begin{abstract}
We study the creation of vortex states in a trapped Bose-Einstein condensate by a rotating force. For a harmonic trapping potential the rotating force induces only a circular motion of the whole condensate around the trap center which does not depend on the interatomic interaction. For the creation of a pure vortex state it is necessary to confine the atoms in an anharmonic trapping potential. The efficiency of the creation can be greatly enhanced by a sinusodial variation of the force's angular velocity. We present analytical and numerical calculations for the case of a quartic trapping potential. The physical mechanism behind the requirement of an anharmonic trapping potential for the creation of pure vortex states is explained.
\end{abstract}

\section{INTRODUCTION}

The experimental realization of magnetically trapped Bose-Einstein condensates (BEC) of alkalimetal atoms [1] has led to a broad interest in the properties of this state of quantum matter. One aspect of particular interest is to study the superfluid behaviour of these systems. An ideal tool to examine this is to rotate the condensate and to observe whether such a rotation can result in the creation of vortex states.

Theoretically many aspects of vortex states in a trapped BEC have been discussed in the literature. The critical angular velocity required to create a vortex state in trapped BECs was derived by Baym and Pethick [2]. Dalfovo and Stringari determined numerically the shape of the vortex state [3] and the moment of inertia in harmonic traps [4]. Ho and Shenoy 5 discussed the influence of the internal atomic structure on vortex states and Barenghi [6] studied vortex waves. Wilkin et al. [7] considered the fragmentation of a rotating BEC with attractive interaction. Dodd et al. [8] have numerically studied excitations around a vortex state. Rokshar showed that the stability of a vortex state depends on the shape of the trap [9] and considered the properties of vortex states on a torus [10].

Despite recent interest in the rotational properties of BECs, the experimental realization of a vortex state remains to be done [11]. In contrast to superfluid Helium, the trapped BECs are not in direct contact to an external container. How to rotate the trapped BECs into vortex states is still an open question. We have recently proposed to transfer angular momentum to a BEC by using multiple travelling wave laser beams 12 and to create vortex states by means of Laguerre-Gaussian laser beams using a Raman transition [13. A related idea based on Raman transitions has also independently been proposed by Bolda and Walls [14]. In addition, a combination of multiple laser beams with Raman transition has recently been employed to discuss the creation of dark solitons and vortices in trapped BECs [15].

In the present paper we study different aspects of the quantized circular motion of a trapped BEC driven by a spatially homogeneous rotating force. In Ref. [12] we showed that such a force can be created by four travelling wave laser beams. In particular, we analyse the reason why in a harmonic trap the rotating force only leads to a rotational state of BECs which is a coherent superposition of vortex states. On this basis, we further demonstrate how to create a pure vortex state by introducing an anharmonic trapping potential.

The paper is organised as follows. In Sec. II we derive the Gross-Pitaevskii equation for trapped BECs composed of two-level atoms in the presence of highly detuned laser fields with different frequencies. In Sec. III, we review the proposal of Ref. [12] and apply the general model to the transfer of angular momentum to BECs by a configuration of four travelling-wave laser beams. The force induced 
by these laser beams is spatially homogeneous and rotates with the frequency difference between the laser beams. In Sec. IV we discuss the center-of-mass motion and the shape of a harmonically trapped BEC driven by the rotating force. Further we analyze the physical mechanism of why rotating a harmonically trapped BEC cannot create a pure vortex state. The possibility to create a pure vortex state of an ideal BEC is demonstrated in Sec. $\mathrm{V}$ by introducing an anharmonic trapping potential. In Sec. VI we consider an interacting Bose gas in an anharmonic trap. With a sinusodial modulation of the frequency difference between the laser beams the nonlinear energy shift due to the interatomic interaction can be compensated. We numerically show that an almost pure vortex state can be created. The conlusion is included in Sec. VII with a short discussion of the relation between the present proposal and that of Refs. [13,14] based on Raman transitions.

\section{GROSS-PITAEVSKII EQUATION FOR A BEC IN MULTIPLE LASER BEAMS}

We consider a condensate of $N$ two-level atoms at zero temperature confined in a trapping potential. In the formalism of quantum field theory this system can be described by the Hamiltonian

$$
H_{0}=\int d^{3} x \sum_{i=e, g}\left\{\Psi_{i}^{\dagger}(\vec{x})\left[H_{c . m .}+E_{i}-\mu\right] \Psi_{i}(\vec{x})\right\}+\frac{1}{2} \int d^{3} x \sum_{i, j=e, g} g_{i j} \Psi_{i}^{\dagger}(\vec{x}) \Psi_{j}^{\dagger}(\vec{x}) \Psi_{j}(\vec{x}) \Psi_{i}(\vec{x})
$$

The symbol $\mu$ denotes the chemical potential. The center-of-mass Hamiltonian

$$
H_{c . m .}:=\frac{-\hbar^{2} \Delta}{2 M}+V_{\text {trap }}(\vec{x})
$$

contains the kinetic energy and the trapping potential $V_{\text {trap }}(\vec{x})$, which is assumed to be cylindrically symmetric around the z-axis. $\Psi_{i}(\vec{x})$ denote the field operator for atoms in the internal ground state $(i=g)$ and excited state $(i=e)$ with internal energy $E_{i}$. $M$ dentoes the atomic mass. Without affecting the generality of our final results we have assumed that the trap potential is the same for both internal states. The second integral in Eq. (11) describes the interatomic interaction in the absence of laser fields. The factors $g_{i j}$ are defined as $4 \pi \hbar^{2} a_{i j} / M$, with $a_{i j}$ being the s-wave scattering lengths for atoms with different internal states.

The interaction of the atoms with the laser beams is incorporated by the electric dipole coupling in the rotating wave approximation,

$$
H_{\text {int }}=-\int d^{3} x\left\{\Psi_{e}^{\dagger}(\vec{x}) \Psi_{g}(\vec{x}) \sum_{a=1}^{n} \hbar \Omega_{a}^{(+)}(\vec{x}) e^{-i \omega_{a} t}+H . c .\right\} .
$$

Here $\omega_{a}, a=1, \ldots, n$ is the frequency of the ath laser beam, and $\Omega_{a}^{(+)}(\vec{x})$ is defined as $\vec{d} \cdot \vec{E}_{a}^{(+)}(\vec{x}) / \hbar$ with $\vec{d}$ being the dipole moment of the atoms and $\vec{E}_{a}^{(+)}$denoting the positive frequency part of the $a$ th laser's electric field.

It has been discussed at length in the literature [16 18 how this Hamiltonian can be reduced to an effective Hamiltonian for atoms being in their internal ground state in the presence of a single laser beam. For this reason we will only briefly discuss how this can be done in the presence of several laser beams with different frequencies. In order to avoid spontaneous emission all laser beams have to be detuned far off resonance so that the population of the excited-state atoms is negligible. In this case the Heisenberg equation of motion for the excited-state field operator can be adiabatically eliminated by assuming that the detunings $\Delta_{a}:=\omega_{a}-\left(E_{e}-E_{g}\right) / \hbar$ of the laser beams from the atomic resonance frequency are all in the same order of magnitude and are the largest characteristic frequencies of the system [16]. The resulting expression for $\Psi_{e}$ is given by

$\Psi_{e}(\vec{x}, t) \approx-\sum_{a} \frac{\Omega_{a}^{(+)} e^{-i \omega_{a} t}}{2\left(\Delta_{a}+i \gamma / 2\right)} \Psi_{g}(\vec{x}, t)+\frac{i}{2} \int d^{3} y L^{*}(\vec{x}-\vec{y}) \sum_{a=1}^{n} \frac{\Omega_{a}^{(+)}(\vec{y}) e^{-i \omega_{a} t}}{\left(\Delta_{a}+i \gamma / 2\right)^{2}} \Psi_{g}^{\dagger}(\vec{y}, t) \Psi_{g}(\vec{y}, t) \Psi_{g}(\vec{x}, t)$.

The Kernel $L(\vec{x}-\vec{y})$ describing the photon-exchange induced dipole-dipole interaction is defined in Ref. [16]. The parameter $\gamma$ denotes the sponataneous emission rate from excited atoms and is assumed 
to be much smaller than the detuning of the laser beams in this paper. The expression (4) for $\Psi_{e}$ can be inserted into the Heisenberg equation for $\Psi_{g}$ thus leading to the effective equation

$$
i \hbar \dot{\Psi}_{g}(\vec{x}, t)=-\left[H_{0}, \Psi_{g}(\vec{x}, t)\right]+V_{L}(\vec{x}, t) \Psi_{g}(\vec{x}, t)+V_{N L}(\vec{x}, t) \Psi_{g}(\vec{x}, t),
$$

with the light-induced linear potential being given by

$$
V_{L}(\vec{x}, t):=\frac{\hbar}{4(\bar{\Delta}+i \gamma / 2)} \sum_{a, b=1}^{n} \Omega_{a}^{(+)}(\vec{x}) \Omega_{b}^{(-)}(\vec{x}) e^{-i\left(\omega_{a}-\omega_{b}\right) t} .
$$

Note that in the denominator we have replaced $\Delta_{a}$ by the average detuning $\bar{\Delta}$ of all lasers. This has to be done for consistency since otherwise $V_{L}$ would be non-Hermitian even in absence of spontaneous emission. Mathematically this can be made because the difference $\Delta_{b}-\Delta_{a}$ between two detunings has to be treated as of higher order in $1 / \bar{\Delta}$. Assuming that the condensate has a coherence length larger than the characteristic distance of the photon-exchange induced dipole-dipole interation, we derive the nonlinear potential

$$
V_{N L}(\vec{x}, t)=g_{e g} \Psi_{g}^{\dagger}(\vec{x}, t) \Psi_{g}(\vec{x}, t) \sum_{a, b=1}^{n} \frac{\Omega_{a}^{(+)}(\vec{x}) \Omega_{b}^{(-)}(\vec{x}) e^{-i\left(\omega_{a}-\omega_{b}\right) t}}{4\left(\bar{\Delta}^{2}+\gamma^{2} / 4\right)}
$$

The strength of this potential depends on the intensity of the applied laser fields. As we will be concerned only with very weak laser beams $V_{N L}$ can safely be neglected in the present case.

To describe the collective evolution of a condensed gas of bosonic atoms we will adopt the mean field approach in Eq. (5). This approximation describes a trapped Bose-Einstein condensate quite well [19] and amounts in replacing the field operator $\Psi_{g}(\vec{x}, t)$ in Eq. (5), after the calculation of the commutator, by a collective wavefunction $\sqrt{N} \psi(\vec{x}, t)$ of all atoms in the condensate, with $\psi$ being normalized to one. This procedure results in the Gross-Pitaevskii equation for $\psi$,

$$
i \hbar \frac{d \psi(\vec{x}, t)}{d t}=\left\{H_{c . m .}+V_{L}(\vec{x}, t)+g N|\psi(\vec{x}, t)|^{2}\right\} \psi(\vec{x}, t) .
$$

For brevity we have introduced $g:=g_{g g}$. This equation determines the nonlinear dynamics of trapped BECs in multiple laser beams and defines the effective Hamiltonian

$$
H=H_{c . m .}+V_{L}+N g|\psi|^{2} \psi
$$

for the collective wavefunction $\psi$.

\section{TRANSFER OF ANGULAR MOMENTUM BY AN OPTICAL POTENTIAL}

The design of an optical potential $V_{L}$ which can rotate the BEC by transferring angular momentum was addressed in Ref. [12] to which we refer for all details of the construction. Here we will only briefly sketch the important features of this optical potential.

The potential $V_{L}(\vec{x}, t)$ of Ref. [12] is created by four traveling wave laser beams of equal intensity and width much larger than the size of the condensate (this is usually the case) so that they can be considered as plane waves. The laser beams 1 and 2 propagate along the $\mathrm{x}$ - and y-direction have the same frequency $\omega_{1}=\omega_{2} \equiv \omega$. Their phases are chosen to be equal at the origin so that the Rabi frequencies of the two lasers have the form $\Omega_{1}^{(+)}(\vec{x})=\Omega_{0} \exp [i k x]$ and $\Omega_{2}^{(+)}(\vec{x})=\Omega_{0} \exp [i k y]$. The other two laser beams have the frequency $\omega_{3}=\omega_{4} \equiv \omega^{\prime}$ and propagate in directions slightly different from the $\mathrm{x}$ - and $\mathrm{y}$ - direction with a small angle $\theta$. The frequency difference $\omega-\omega^{\prime}$ is assumed to be so small that the difference between $\left|\vec{k}_{a}\right|$ and $\left|\vec{k}_{b}\right|$ for any $a, b,=1, \ldots, 4$ is negligible. The wavevector of the third laser beam is given by $k \cos (\theta) \vec{e}_{x}-k \sin (\theta) \vec{e}_{y} \approx k \vec{e}_{x}-\delta k \vec{e}_{y}$, where

$$
\delta k:=k \theta
$$

denotes the deviation of the wavevectors. Similiarly the 4 th laser's wavevector is given by $k \vec{e}_{y}-$ $\delta k \vec{e}_{x}$. The phases are chosen so that laser 4 is in phase with lasers 1 and 2 at the origin whereas 
the phase of laser 3 is shifted by $-\pi / 2$. The third Rabi frequency is then given by $\Omega_{3}^{(+)}(\vec{x})=$ $\exp [-i \pi / 2] \Omega_{0} \exp [i(k x-\delta k y)]$ and the fourth by $\Omega_{4}^{(+)}(\vec{x})=\Omega_{0} \exp [i(k y-\delta k x)]$.

The described configuration of laser beams leads to the optical potential

$$
\begin{aligned}
V_{L}(\vec{x}, t)= & \hbar \Omega_{\mathrm{eff}}\left\{e^{-i k(x-y)}\left[1+i e^{-i \delta k(x-y)}\right]\right. \\
& \left.+e^{-i \phi(t)}\left[e^{i \delta k x}+i e^{i \delta k y}+i e^{-i k(x-y)+i \delta k y}+e^{i k(x-y)+i \delta k x}\right]\right\}+ \text { H.c. },
\end{aligned}
$$

where we have introduced the effective Rabi frequency $\Omega_{\text {eff }}:=\left|\Omega_{0}\right|^{2} /(4 \bar{\Delta})$ and the time dependent phase $\phi(t):=t\left(\omega-\omega^{\prime}\right)$. In this section and in the rest of the paper we neglect c-number terms in the optical potential since they only produce an overall phase shift in the wavefunction.

Since the wavelength of an optical laser is much smaller than the size of a typical condensate (several micrometers) all exponentials in Eq. (11) containing the wavevector $k$ are rapidly varying over the condensate and therefore average to zero [12]. The remaining exponentials, $\exp [i \delta k x]$ and $\exp [i \delta k y]$, can be approximated by expressions linear in $\delta k$ if the angle $\theta$ is small enough. The resulting optical potential then takes the form.

$$
V_{L}(\vec{x}, t)=2 \hbar \Omega_{\mathrm{eff}} \delta k\{\sin (\phi(t)) x-\cos (\phi(t)) y\} .
$$

It obviously produces a rotating spatially homogeneous force over the size of the condensate. The rotating force can transfer angular momentum to the condensate. This can be seen easily by rewriting it in the form

$$
V_{L}(\vec{x}, t)=i \hbar \Omega_{\mathrm{eff}} \delta k\left\{(x+i y) e^{-i \phi(t)}-(x-i y) e^{i \phi(t)}\right\} .
$$

Because of the commutation relation $\left[L_{z},(x \pm i y)\right]= \pm \hbar(x \pm i y)$ the operator $x+i y$ increases the orbital angular momentum by $\hbar$. Thus, if the frequency difference $\omega-\omega^{\prime}$ is nearly resonant with a transition between two trap eigenstates, the angular momentum of the condensate is increased by the force [12].

Since $V_{L}$ is produced by laser beams we have to estimate the rate of decoherence of the condensate due to the spontaneous emission of photons which is given by the probability that an atom is excited times the spontaneous emission rate $\gamma$. The excitation probability for off-resonant light is of the order of $\left|\Omega_{0}\right|^{2} / \bar{\Delta}^{2}=\Omega_{\text {eff }} / \bar{\Delta}$. The decoherence time is therefore given by $\bar{\Delta} /\left(\gamma \Omega_{\text {eff }}\right)$ and is therefore much larger than $1 / \Omega_{\text {eff }}$ since the detuning is much larger than the natural linewidth. For instance, if $\left.1 / \Omega_{\text {eff }}\right)$ is about $10 \mathrm{~ms}$ then for $\bar{\Delta}=100 \gamma$ the decoherence time is in the order of a second.

One also has to address the question of how stable the frequencies of the laser beams must be so that $\omega-\omega^{\prime}$ is well defined. It turns out that this poses no problem at all since only the frequency difference $\omega-\omega^{\prime}$ is required to be stable. Since in the proposal of Ref. 12 all four laser beams originate from the same laser source, and since the frequency difference is created by modulating the laser beams, it is clear that only the modulator frequency must be stable. The stability requirement on the laser beams can therefore easily be fulfilled by any common laser source.

We also point out that laser beams are of course only one possibility to produce a rotating homogeneous force. The same potential could be created by spatially varying and rotating magnetic fields, for instance. The following analysis of the condensate's motion in the potential (13) therefore covers this case as well.

\section{HARMONICALLY TRAPPED CONDENSATE: COHERENT ROTATION}

We now turn to the effect of the optical potential (13) on a condensate trapped in a harmonical potential of the form

$$
V_{\text {trap }}(\vec{x})=\frac{M}{2} \omega_{z}^{2} z^{2}+\frac{M}{2} \omega_{\perp}^{2}\left(x^{2}+y^{2}\right) .
$$

$\omega_{z}$ and $\omega_{\perp}$ denote the trap frequency in the z-direction and in the $\mathrm{x}$-y-plane, respectively.

We have shown in Ref. 12] that in the case of an ideal Bose gas and a resonant optical potential

(i.e., $\omega-\omega^{\prime}=\omega_{\perp}$ ) the condensate rotates around the center of the trap with increasing mean radius 
but preserving its shape (comp. Fig. 1). It was also demonstrated that this motion corresponds to a coherent superposition of vortex states $\psi_{n}(\propto \exp [i n \varphi]$, where $\varphi$ is the azimuthal angle in the $\mathrm{x}$-y-plane) with different winding number $n$.

In this section we extend this result to an interacting Bose gas. We start from Eq. (9) including the resonant optical potential (13). As is well-known the time evolution of the expectation value of any operator $O$ is governed by the equation

$$
\frac{d}{d t}\langle O\rangle=\frac{i}{\hbar}\langle[H, O]\rangle
$$

It is straightforward to derive from this relation the corresponding equations for the position operators. Observing that $\int d^{3} x|\psi|^{2} \partial_{i}|\psi|^{2}, i=x, y, z$ vanishes the center-of-mass motion takes the simple form

$$
\frac{d^{2}}{d t^{2}}\langle\xi\rangle+\omega_{\xi}^{2}\langle\xi\rangle=f_{\xi}(t)
$$

for $(\xi=x, y, z)$, where the light-induced force $\vec{f}(t)$ is given by $\vec{f}(t)=2\left(\hbar \Omega_{\text {eff }} \delta k / M\right)\left\{-\sin \left(\omega_{\perp} t\right) \vec{e}_{x}+\right.$ $\left.\cos \left(\omega_{\perp} t\right) \vec{e}_{y}\right\}$. For a BEC in the ground state the condensate's mean position and mean momentum are initially zero $(\langle\vec{x}\rangle(0)=0,\langle\vec{p}\rangle(0)=0)$ so that we find for the solution

$$
\langle\vec{x}\rangle=R_{0}\left\{\vec{e}_{x}\left[\omega_{\perp} t \cos \left(\omega_{\perp} t\right)-\sin \left(\omega_{\perp} t\right)\right]+\vec{e}_{y} \omega_{\perp} t \sin \left(\omega_{\perp} t\right)\right\}
$$

with $R_{0}:=\hbar \Omega_{\mathrm{eff}} \delta k /\left(M \omega_{\perp}^{2}\right)$. Again the condensate performs a rotation around the trap center whereby the radius of the rotation increases linearly in time. This result was to be expected since usual interatomic potentials are translation-invariant and therefore affect the center-of-mass motion only indirectly. It provides an extension of Ehrenfest's theorem for interacting Bose condensates. We remark that the result (17) deviates from the corresponding result of Ref. [12] by the factor $-\sin \left(\omega_{\perp} t\right)$ inside the square brackets. This difference arises because in Ref. [12] a rotating wave approximation (RWA) has been made. As usual for RWA the difference is negligible for large times $\omega_{\perp} t \gg 1$.

To demonstrate that, as in the noninteracting case, the shape of the condensate is not affected by the optical potential we consider the time evolution of the variance of the position operators. Using $\int d^{3} x \xi|\psi|^{2} \partial_{\xi}|\psi|^{2}=-\int d^{3} x|\psi|^{4} / 2$ and defining $\Lambda_{\xi}:=\left\langle\xi p_{\xi}+p_{\xi} \xi\right\rangle-2\langle\xi\rangle\left\langle p_{\xi}\right\rangle$ we arrive at

$$
\begin{aligned}
\frac{d(\Delta \xi)^{2}}{d t} & =\frac{\Lambda_{\xi}}{M} \\
\frac{d \Lambda_{\xi}}{d t} & =\frac{2}{M}\left(\Delta p_{\xi}\right)^{2}-2 M \omega_{\xi}^{2}(\Delta \xi)^{2}+g \int d^{3} u|\psi(\vec{u})|^{4} \\
\frac{d\left(\Delta p_{\xi}\right)^{2}}{d t} & =-M \omega_{\xi}^{2} \Lambda_{\xi}-i \hbar g \int d^{3} u\left\{\left(\partial_{\xi} \psi^{*}(\vec{u})\right)^{2} \psi^{2}(\vec{u})-H . c .\right\} .
\end{aligned}
$$

Because neither the center-of-mass motion nor the optical potential enter into these equations we can infer that the shape of the condensate remains unaffected by the optical potential.

It is worth noting that one can derive from Eq. (18) the mean energy conservation law

$$
\frac{1}{2 M}(\Delta \vec{p})^{2}+\frac{1}{2} M\left\{\omega_{\perp}^{2}\left[(\Delta x)^{2}+(\Delta y)^{2}\right]+\omega_{z}^{2}(\Delta z)^{2}\right\}+g \int d^{3} u|\psi(\vec{u})|^{4}=\text { const. }
$$

which nicely demonstrates the relation between the interaction energy and the widths of the condensate's wavefunction.

We conclude that, albeit the potential (13) seems to be perfectly suited for this task, neither a pure vortex state nor even a vortex line far away from the center of the trap is created. However, this does not rule out the possibility to form a vortex state during the formation of the condensate by directly cooling the gas in a rotating trapping potential. Our result may also be helpful to understand why the recent experiments at MIT [11] attempting to observe persistent flows around a potential wall at the trap center by rotating the trap cannot achieve the expected result.

To understand why the potential (13) produces a superposition of vortex states rather than a pure one we observe that it couples only neighboring eigenstates of the trap. This can be seen by expressing the coordinates $x, y$ in terms of the corresponding annihilation operators of the trap (i.e., $x \propto a_{x}+a_{x}^{\dagger}$, for instance). Since the potential is linear in these operators it couples states with energy $n \hbar \omega_{\perp}$ (in 
the noninteracting case) to states with energy $(n+1) \hbar \omega_{\perp}$. If the coupling is resonant $\left(\phi(t)=\omega_{\perp} t\right)$ the potential (13) not only resonantly transforms the ground state to the first excited vortex state, but it also resonantly transfers the $n$th vortex state (with energy $n \hbar \omega_{\perp}$ ) to the $(n+1)$ th vortex state, because of the equal energy interval between these energy eigenstates. As a result, the long-time evolution of the system driven by the rotating force will lead to a superposition of vortex states with different winding number.

This implies that to create a pure vortex state one must destroy the equal interval between the trap energy levels to avoid the simultaneous excitations of different vortex states. We will analyze this in the next section

\section{CREATION OF A VORTEX STATE IN AN IDEAL BOSE GAS}

In the preceding sections we have presented a scheme to transfer a harmonically trapped condensate into a rotating state which is a coherent superposition of vortex states. We also analysed why the scheme does not create a pure vortex state if the trapping potential is harmonic: the equal interval between the energy levels in a harmonic trap causes the condensate to climb up a ladder of resonant transitions into different vortex states.

The situation is different if an anharmonic (yet still rotationally symmetric) potential $V_{\text {trap }}$ is used in Eq. (8) for which the interval between the energy levels is not equal. Therefore, if the frequency $\omega-\omega^{\prime}$ in the optical potential $(13)$ is choosen to be in resonance with the transition from the ground state to the first excited vortex state, the transition to the next vortex state will in general be out of resonance (see Fig. 2). This fact can be exploited to prepare a pure vortex state in an anharmonic trap.

To estimate this quantitatively we first consider the ideal Bose gas and expand the wavevector in terms of the simultaneous eigenstates $\psi_{m, s}$ of the Hamiltonian (2) and the orbital angular momentum $J_{z}, \psi=\sum_{m, s} \exp [-i m \phi(t)] c_{m, s}(t) \psi_{m, s}$. The eigenstates fulfill the relations $H_{c . m .} \psi_{m, s}=E_{m, s} \psi_{m, s}$ and $J_{z} \psi_{m, s}=m \hbar \psi_{m, s}$. It should be noted that the inclusion of the phase factor $\exp [-i m \phi(t)]$ in this expansion is equivalent to the description of the system in a frame of reference rotating with angular velocity $\dot{\phi}(t)=\omega-\omega^{\prime}$ (where we left open the possibility that the frequencies can vary in time). In this description the broken symmetry between states of positive $(m>0)$ and negative $(m<0)$ angular momentum becomes obvious. Since the latter are counterrotating for $\omega-\omega^{\prime}>0$ transitions to these states are highly suppressed.

Using this expansion the Schrödinger equation (8) for the ideal condensate with $g=0$ can be reduced to

$$
i \hbar \dot{c}_{m, s}=\left\{E_{m, s}-m \hbar \dot{\phi}(t)\right\} c_{m, s}+\hbar \Omega_{\mathrm{eff}} \delta k \sum_{s^{\prime}}\left\{q_{m ; s, s^{\prime}} c_{m-1, s^{\prime}}+q_{m+1 ; s^{\prime}, s}^{*} c_{m+1, s^{\prime}}\right\}
$$

with $q_{m ; s, s^{\prime}}:=\int d^{2} x \psi_{m, s}^{*}(x+i y) \psi_{m-1, s^{\prime}}$.

We assume that, as it is the case for the harmonic potential, $H_{c . m}$. has a unique ground state with energy $E_{0,0}$ and that the energy levels $E_{1, s}$ are well separated, with $E_{1,0}$ being the lowest vortex excitation. In this case, if we choose $\omega-\omega^{\prime}=\left(E_{1,0}-E_{0,0}\right) / \hbar$ the transition from $\psi_{0,0}$ to $\psi_{1,0}$ is in resonance while the transition from $\psi_{1,0}$ to $\psi_{2, s}$ is detuned by the amount $\Delta_{s}:=\omega-\omega^{\prime}-\left(E_{2, s}-E_{1,0}\right)=$ $E_{2, s}+E_{0,0}-2 E_{1,0}$. Furthermore, if the condition $\left|\Omega_{\mathrm{eff}} \delta k q_{2 ; s, 0}\right| \ll \Delta_{s}$ is fulfilled, the transition from $\psi_{1,0}$ to $\psi_{2, s}$ will be highly supressed so that we effectively have a two-level system consisting of the states $\psi_{0,0}$ and $\psi_{1,0}$. It is obvious that after a time $\pi / \nu$ the complete population will have been transferred to the first excited vortex state, where we have defined the Rabi frequency

$$
\nu:=\left|\Omega_{\mathrm{eff}} \delta k q_{1 ; 0,0}\right|
$$

of the transition between $\psi_{0,0}$ and $\psi_{1,0}$ This situation is mathematically very similar to the resonant excitation of the first excited state in an atom by a laser beam. In this case one can safely neglect all states beside the ground and the first excited state, too.

As a specific example we consider a two-dimensional anharmonic oscillator for which the trapping potential is given by $V_{\text {trap }}=\kappa r^{4}$, where $r$ is the two-dimensional radial variable and $\kappa$ determines the strength of the potential. The neglection of the $\mathrm{z}$-direction does not alter the predictions for the ideal Bose gas and is justified for the interacting Bose gas if excitations along the z-axis are sufficiently 
supressed. Approximate eigenstates of the resulting Hamiltonian can be obtained by application of Ritz's variational method. For later use we include the nonlinearity and minimize the expression

$$
E=\int d^{2} x\left\{\psi^{*}\left[\frac{\vec{p}^{2}}{2 M}+\kappa r^{4}\right] \psi+\frac{g_{2 D}}{2}|\psi|^{4}\right\}
$$

for the conserved energy. The coupling constant $g_{2 D}$ that we use for an effective description of the vortex creation in two dimensions is related to the three-dimensional coupling parameter $g$ by $g_{2 D}=g N / l_{0}$. Using the ansatz

$$
\psi_{n, 0}(\vec{x})=\sqrt{\frac{\alpha_{n}^{n+1}}{\pi n !}} r^{n} e^{i n \varphi} e^{-\alpha_{n} r^{2} / 2} .
$$

we find for the variational energy eigenvalues the expression $E_{n, 0}=E_{n, 0}^{(L)}+E_{n, 0}^{(N L)}$, with

$$
E_{n, 0}^{(L)}=\mathcal{E} \frac{(n+1)}{2}\left(3+\mathcal{G} f_{n}\right)\left[\frac{2(n+2)}{1+\mathcal{G} f_{n}}\right]^{1 / 3} \quad, \quad E_{n, 0}^{(N L)}=\mathcal{E} \mathcal{G} f_{n}(n+1)\left[\frac{2(n+2)}{1+\mathcal{G} f_{n}}\right]^{1 / 3}
$$

$E_{n, 0}^{(L)}$ represents the contribution of the linear part of the Hamiltonian (kinetic and potential energy) and $E_{n, 0}^{(N L)}$ denoted the contribution of the nonlinear interaction energy. We have introduced the energy scale $\mathcal{E}:=\kappa l_{0}^{4}$, the dimensionless interaction parameter $\mathcal{G}:=M g_{2 D} /\left(2 \pi \hbar^{2}\right)$, and the numerical factor $f_{n}:=(2 n-1) ! ! /\left[(n+1) ! 2^{n}\right]$. The characteristic length scale of the system is given by $l_{0}:=$ $\left[\hbar^{2} /(2 M \kappa)\right]^{1 / 6}$. For the ideal Bose gas we set $\mathcal{G}=0$ and find for the lowest energy eigenvalues the values $E_{0,0} \approx 2.38 \cdot \mathcal{E}, E_{1,0} \approx 5.45 \cdot \mathcal{E}, E_{2,0} \approx 9 \cdot \mathcal{E}$, and $E_{0,1} \approx 9.64 \cdot \mathcal{E}$. We have determined the energy $E_{0,1}$ by using the ansatz $\psi_{0,1} \propto\left(\varepsilon^{2}-r^{2}\right) e^{-\delta r^{2} / 2}$ for the wavefunction. The largeness of $E_{0,1}$ allows us to neglect all transitions to this state.

The interaction matrix elements are of the order of $l_{0}$ for these four states, but we will need only the value for $q_{1 ; 0,0} \approx 0.79 \cdot l_{0}$ and $q_{2 ; 0,0} \approx 1.05 \cdot l_{0}$. The transition from $|0,0\rangle$ to $|1,0\rangle$ will be in resonance if we choose the frequency difference $\dot{\phi}=3.07 \cdot \mathcal{E} / \hbar$. The transition from $|1,0\rangle$ to $|2,0\rangle$ is then supressed if the interaction matrix element $\hbar \Omega_{\mathrm{eff}} \delta k \cdot q_{2 ; 0,0}$ is much smaller than the energy difference $E_{2,0}-E_{1,0}-\hbar \dot{\phi} \approx 0.48 \cdot \mathcal{E}$. This is guaranteed if we set the transition energy $E_{\text {trans }}:=\hbar \nu$ equal to $\mathcal{E} / 20$. The laser scheme presented above then will create a vortex state after a time $\pi / \nu$. We have depicted this physical situation (to scale) in Fig. 3.

To estimate its experimental realizability and to compare it with the interacting Bose gas we consider the case that the numerical value of the trapping potential strength $\kappa$ is given by $7.66 \cdot 10^{-8} \mathrm{~J} / \mathrm{m}^{4}$ so that for ${ }^{87} \mathrm{Rb}$ atoms $\left(M=1.45 \cdot 10^{-25} \mathrm{~kg}\right.$ ) the trap size turns out to be $l_{0}=8.91 \cdot 10^{-7} \mathrm{~m}$ (These numerical values turn out to be convenient for the numerical calculations presented below). We then find for the energy scale $\mathcal{E} \approx 4.83 \cdot 10^{-32} \mathrm{~J}$ and for the characteristic time for vortex creation $\pi / \nu \approx 70$ ms. This well within the range of current experiments with Bose-Einstein condensates. We mention that we have also made a numerical study of this creation process for the numbers given here. The result is in excellent agreement with our analytical calculations but will not be presented here since the analytical results already perfectly describe the physical situation for a non-interacting Bose gas. For all details of the numerical procedure we refer to the next section where it is applied to the correponding case of an interacting Bose gas.

\section{CREATION OF A VORTEX STATE IN AN INTERACTING BOSE GAS}

To study the influence of the atomic interaction analytically we assume that the nonlinearity in Eq. (8) is weak enough so that we still can use the variational ansatz (23). This is not allowed for the strong interaction regime where this ansatz becomes meaningless, but it includes the case that the interaction energy is of the order of the kinetic and potential energy and thus overcomes the restriction of perturbation theory.

We further assume that radial excitations of the condensate are unimportant because the energy levels $E_{m, s}(s>0)$ are considerably larger than the corresponding lowest value $E_{m, 0}$ for the same angular momentum $m \hbar$. We then only need to take into account the states $\psi_{m, 0}$. The 
numerical results presented below further justify this assumption. Writing the wavefunction as $\psi=\sum_{n} c_{n}(t) \exp [-i n \Delta \omega t] \psi_{n, 0}(\vec{x})$ and projecting Eq. (8) onto $\psi_{m, 0}$ we arrive at

$$
\begin{aligned}
i \hbar \dot{c}_{m}= & \left\{E_{m, 0}^{(L)}-m \hbar \dot{\phi}\right\} c_{m}+\hbar \Omega_{\mathrm{eff}} \delta k\left\{q_{m ; 0,0} c_{m-1}+q_{m+1 ; 0,0}^{*} c_{m+1}\right\} \\
& +2 E_{m, 0}^{(N L)}\left|c_{m}\right|^{2} c_{m}+\sum_{k \neq m} E_{m, k}^{(S)}\left|c_{k}\right|^{2} c_{m}+\sum_{l \neq m} \sum_{k \neq l} c_{k}^{*} c_{l} c_{m+k-l} E_{m ; k, l}^{(A S)} .
\end{aligned}
$$

Here we have introduced the energies $E_{m, k}^{(S)}=E_{k, m}^{(S)}:=2 g_{2 D} \int d^{2} x\left|\psi_{m, 0}\right|^{2}\left|\psi_{k, 0}\right|^{2}$ for scattering between atoms in state $k$ and $m$, and $E_{m ; k, l}^{(A S)}:=g_{2 D} \int d^{2} x \psi_{m, 0}^{*} \psi_{k, 0}^{*} \psi_{l, 0} \psi_{m+k-l, 0}$ for anomalous scattering of atoms.

To solve Eq. (25) analytically we first make the assumption that only the two states $\psi_{0,0}$ and $\psi_{1,0}$ are populated. Though this is not justified for a quantitative analysis it provides very useful physical insight into the effectiveness of the process of vortex creation. Eq. (25) then reduces to

$$
\begin{aligned}
& i \hbar \dot{c}_{0}=\left\{E_{0,0}^{(L)}+2 E_{0,0}^{(N L)}\left|c_{0}\right|^{2}+E_{0,1}^{(S)}\left|c_{1}\right|^{2}\right\} c_{0}+\hbar \Omega_{\mathrm{eff}} \delta k q_{1 ; 0,0}^{*} c_{1} \\
& i \hbar \dot{c}_{1}=\left\{E_{1,0}^{(L)}+2 E_{1,0}^{(N L)}\left|c_{1}\right|^{2}+E_{0,1}^{(S)}\left|c_{0}\right|^{2}-\hbar \dot{\phi}\right\} c_{1}+\hbar \Omega_{\mathrm{eff}} \delta k q_{1 ; 0,0} c_{0} .
\end{aligned}
$$

This form of the nonlinear Schrödinger equation is particularly suited to study the effect of a timedependent variation of the laser frequencies $\dot{\phi}=\omega-\omega^{\prime}$. It was originally proposed in Ref. [13] to use such a variation to compensate for the nonlinear interaction energy between the atoms. Here we will go into more detail and examine the question of which variation is the most effective one to create a vortex state.

Let us first assume that $\dot{\phi}$ is constant and chosen so that initially $\left(\left|c_{0}\right|^{2}=1,\left|c_{1}\right|^{2}=0\right)$ the transition from $\psi_{0,0}$ to $\psi_{1,0}$ is on resonance, i.e.,

$$
\dot{\phi}=E_{1,0}^{(L)}-E_{0,0}^{(L)}+E_{1,0}^{(S)}-2 E_{0,0}^{(N L)} .
$$

After some time the population of the two states will be altered $\left(\left|c_{0}\right|^{2}<1,\left|c_{1}\right|^{2}>0\right)$ so that the magnitude of the terms in curly brackets in Eqs. (26, 27) is changed. Thus, the 2-level system is driven out of resonance by the nonlinear atomic interaction and a complete transfer of the condensate from $\psi_{0,0}$ to $\psi_{1,0}$ becomes impossible.

It is not hard to see that a time-dependent variation of $\dot{\phi}$ can compensate for this interactioninduced shift of the resonance frequency. In Ref. [13] we proposed to vary $\omega-\omega^{\prime}$ linearly in time. While this works well if the rate of change is suitably chosen, it has the disadvantage that one needs a relatively long time to create a vortex state. This is similar to a Landau-Zener transition 20,21 which is destroyed by nonadiabatic transitions if the frequency's rate of change is too high (see Ref. 22, for instance). To improve this situation we assume that $\dot{\phi}$ is implicitely chosen so that the transition between $\psi_{0,0}$ and $\psi_{1,0}$ is always on resonance,

$$
\dot{\phi}=E_{1,0}^{(L)}-E_{0,0}^{(L)}+E_{1,0}^{(S)}\left(\left|c_{0}\right|^{2}-\left|c_{1}\right|^{2}\right)+2 E_{1,0}^{(N L)}\left|c_{1}\right|^{2}-2 E_{0,0}^{(N L)}\left|c_{0}\right|^{2} .
$$

Under this condition the solution of Eqs. 26, 27) is given by

$$
\begin{aligned}
& c_{0}(t)=e^{-i \theta(t)} \cos (\nu t) \\
& c_{1}(t)=\frac{-i q_{1 ; 0,0}}{\left|q_{1 ; 0,0}\right|} e^{-i \theta(t)} \sin (\nu t),
\end{aligned}
$$

where the phase factor $\theta(t)$ is easy to calculate but not needed here. This solution corresponds to a resonant Rabi Oscillation between the two states. Hence the two-level model predicts that the creation of a vortex state can be as effective as in the linear case provided the nonlinear interaction energy is compensated for. The corresponding variation of the frequency difference $\dot{\phi}$ can be found by reinserting Eq. (30) into Eq. (29) and turns out to be a simple sinusodial variation with frequency $2 \nu$. Such a sinusodial variation of the frequency difference $\dot{\phi}$ can be realized by simple frequency modulation techniques.

The above analysis was made under the oversimplified assumption that we only need two levels to take into account. The influence of other modes can be studied in several ways. For instance, they 
can be treated as an elementary excitation around the solution (30). For the sake of brevity we will not discuss the analytical treatment here and present instead numerical solutions of the full nonlinear Schrödinger equation (8).

Numerical solution: Our numerical procedure started from the ground state of the interacting condensate in the quartic anharmonic potential. This state was found by propagating a Gaussian trial wavefunction in imaginary time and normalizing it between each step. The formation of a vortex state was studied by using the modified split-step method presented in Ref. 23. This consists in approximating the time evolution operator $\exp [-i \Delta t(T+V) / \hbar]$ for small $\Delta t$ as $\exp [-i \Delta t V) /(2 \hbar)] \exp [-i \Delta t T \hbar] \exp [-i \Delta t V /(2 \hbar)]$, where $T$ and $V$ denote the kinetic and potential part of the Hamiltonian, respectively. The terms containing $V$ are diagonal in position space and can easily be applied to the wavefunction. The term involving $T$ is computed by transforming the wavefunction to momentum space by using the fast-Fourier-transformation algorithm (FFT), applying the operator, and performing the inverse FFT.

The physical parameters for the trap and the $\mathrm{Rb}$ atoms are the same as given at the end of the previous section (trap strength $\kappa=7.66 \cdot 10^{-8} \mathrm{~J} / \mathrm{m}^{4}$, trap size $l_{0}=8.91 \cdot 10^{-7} \mathrm{~m}$, mass $M=1.45 \cdot 10^{-25}$ $\mathrm{kg}$ ). In addition, the two-dimensional nonlinearity parameter $g_{2 D}$ was assumed to be $2.6 \cdot 10^{-43} \mathrm{~J} \mathrm{~m}^{2}$. For $\mathrm{Rb}$ atoms the scattering length is about $5 \mathrm{~nm}$. Therefore, if we relate an effective 3-dimensional peak density $\hat{\rho}_{3 D}$ to the peak of the 2-dimensional wavefunction $\hat{\psi}_{2 D}$ by $\left|\hat{\psi}_{2 D}\right|^{2} g_{2 D}=\hat{\rho}_{3 D} g$ we can infer that this choice of $g_{2 D}$ corresponds to a peak density $\hat{\rho}_{3 D}$ of about $2 \cdot 10^{13} \mathrm{~cm}^{-3}$. The strength of the optical potential (13) was determined by setting $\hbar \Omega_{\text {eff }} \delta k=2 \cdot 10^{-27} \mathrm{~J} / \mathrm{m}$. It turned out that for this system a grid size of $64 \times 64$ points was accurate enough for the numerical study of the creation process.

The most laborious part of the numerical simulation was to find the proper variation of the frequency difference $\dot{\phi}$. It is not difficult to find an approximation of the physical parameters in Eq. (29) by use of the variational wavefunctions (23). However, in order to get satisfactory results, these parameters must be determined more precise than the variationally calculated values which deviate from the exact values by up to $10 \%$. We finally found that the best results are produced if the sinusodial variation is delayed for a time $t_{0}=50 \mathrm{~ms}$ to compensate for effects of other modes than $\psi_{0,0}$ and $\psi_{1,0}$. Thus the frequency difference was given by

$$
\dot{\phi}=\left\{\begin{array}{cc}
\omega_{\max } & t \leq t_{0} \\
\frac{1}{2}\left\{\left(\omega_{\max }+\omega_{\min }\right)+\left(\omega_{\max }-\omega_{\min }\right) \cos \left(\mu\left(t-t_{0}\right)\right)\right\} & t \geq t_{0}
\end{array},\right.
$$

with $\omega_{\max }=1430 \mathrm{~s}^{-1}, \omega_{\min }=1205 \mathrm{~s}^{-1}$, and $\mu=19.5 \mathrm{~s}^{-1}$.

The numerical results for this choice of parameters are shown in Fig. 4 and 5. Figs. 4a-d show the modulus squared of the wavefunction at different times of the evolution ( $0 \mathrm{~ms}, 87 \mathrm{~ms}, 142 \mathrm{~ms}, 247 \mathrm{~ms})$. It shows that for a moderately interacting Bose gas in an anharmonic trap an almost pure vortex state can be created. Fig. 5 displays the phase of the wavefunction at the same times (the coarse-grained structure at the border of the figures represents numerical noise). Fig. 5b demonstrates that during the evolution two vortex lines are created, one of which finally becomes the vortex state in Fig. $4 \mathrm{~d}$ and the other slips out of the trap. This situation can be described reasonably well by assuming that the wavefunction is a superposition of the form $\psi=c_{0} \psi_{0,0}+c_{1} \psi_{1,0}+c_{2} \psi_{2,0}$, where $\psi_{2,0}$ describes a vortex state with angular momentum $2 \hbar$. Fig. $4 \mathrm{~b}$ shows the corresponding deformation of the wavefunction. The modulus of the expansion coefficients $c_{i}$ can be approximately determined by looking at Fig. 5 since the closer one (two) vortex lines are to the origin the larger the value of $c_{1}\left(c_{2}\right)$ is. We also mention that the vortex lines rotate around the origin during the creation process. This can be seen in an animation of the creation process [24].

\section{CONCLUSION}

We have presented a scheme based on four laser beams which induces a spatially homogeneous force to transfer angular momentum to a trapped condensate. For a harmonic trap this scheme is equivalent to a rotation of the trapping potential around its original position. In this case the condensate will be prepared into a superposition of pure vortex states, corresponding to a circular motion of the condensate as a whole around the original trap center. The reason for the creation of a superposition rather than a pure vortex state is that the energy eigenvalues of the trap are equidistant for a harmonic trap. Thus, transitions to neighboring states are simultaneously resonant. In an anharmonic trap this 
phenomenon does not occur. If the intensity of the laser beam is sufficiently low one can tune the transition from the ground state to the first excited vortex state into resonance while leaving the transition to the second excited vortex state far off-resonant. The interaction-induced energy shift appearing in a non-ideal BEC can be compensated by a sinusodial variation of the rotation frequency of the force. It then is possible to create a pure vortex state in an anharmonic trap.

Beside employing an anharmonic trap one alternative method to create a vortex state is to use Raman transitions induced by Laguerre-Gaussian laser beams, as it was propsed in Refs. [13,14]. In this case a single Laguerre-Gaussian laser beam already carries an orbital angular momentum which can be transferred to the condensate. It is of interest to understand why the proposal with Raman transitions also works for a harmonic trapping potential and is not restricted to anharmonic potentials. In the notation used in the present paper the basic mechanism of the scheme based on Raman-transitions is that laser beams of the Laguerre-Gaussian type couple the condensate, which is initially in the ground state $|0,0\rangle$ of the trap and the internal state $|-\rangle$, to a vortex state $|1,0\rangle$ and the internal state $|+\rangle$. Here the state vectors are defined by $\psi_{m, s}(\vec{x})=\langle\vec{x} \mid m, s\rangle$. The interaction Hamiltonian is then roughly of the form $V_{L} \propto|1,0\rangle \otimes|+\rangle\langle-|\otimes\langle 0,0|+| 0,0\rangle \otimes|-\rangle\langle+| \otimes\langle 1,0|$. This combination of internal transition and excitation of the center-of-mass motion guarantees that the condensate never can occupy higher vortex states like $|2,0\rangle \otimes| \pm\rangle$ since the condensate can only oscillate between the states $|0,0\rangle \otimes|-\rangle$ and $|1,0\rangle \otimes|+\rangle$ in the Raman coupling. Hence selective excitations of the internal state by Raman transitions avoid the resonant excitation of the ladder of transitions to higher vortex states.

Acknowledgement: We thank E.M. Wright and E. Bolda for helpful discussions. The work has been supported by the Australian Research Council.

[1] M. Anderson, J. R. Ensher, M. R. Matthews, C. E. Wieman, and E. A. Cornell, Science 269, 198 (1995); C. C. Bradley, C. A. Sackett, J. J. Tollet and R. Hulet, Phys. Rev. Lett.75, 1687 (1995); M.-O. Mewes, M. R. Andrews, N. J. van Druten, D. M. Kurn, D. S. Durfee, C. G. Townsend and W. Ketterle, Phys. Rev. Lett. 77, 416 (1996); D. S. Jin, J. R. Ensher, M. R. Matthews, C. E. Wieman, and E. A. Cornell, Phys. Rev. Lett. 77, 420 (1996).

[2] G. Baym and C.J. Pethick, Phys. Rev. Lett. 76, 6 (1996).

[3] F. Dalfovo and S. Stringari, Phys. Rev. A 53, 2477 (1996).

[4] S. Stringari, Phys. Rev. Lett. 76, 1405 (1996).

[5] T.-L. Ho and V.B. Shenoy, Phys. Rev. Lett. 77, 2595 (1996).

[6] C.F. Barenghi, Phys. Rev. A 54, 5445 (1996).

[7] N.K. Wilkin, J.M.F. Gunn, and R.A. Smith, preprint cond-mat/9705050.

[8] R.J. Dodd, K. Burnett, M. Edwards, and C.W. Clark, Phys. Rev. A 56, 587 (1997).

[9] D.S. Rokshar, Phys. Rev. Lett. 79, 2164 (1997).

[10] D.S. Rokshar, preprint cond-mat/9709212.

[11] W. Ketterle, talk given at the conference on Bose-Einstein condensation, Castelvecchio Pascoli, Italy, July 1997.

[12] K.-P. Marzlin and W. Zhang, preprint cond-mat/9707294, to appear in Phys. Rev. A.

[13] K.-P. Marzlin, W. Zhang, and E.M. Wright, Phys. Rev. Lett. 79, 4728 (1997).

[14] E. Bolda and D. Walls, preprint cond-mat/9708189.

[15] R. Dum, J.I. Cirac, M. Lewenstein, and P. Zoller, preprint cond-mat/9710238.

[16] W. Zhang and D.F. Walls, Phys. Rev. A 49, 3799 (1994).

[17] G. Lenz, P. Meystre, and E.M. Wright, Phys. Rev. A 50, 1681 (1994).

[18] M. Lewenstein, L. You, J. Cooper, and K. Burnett, Phys. Rev. A 50, 2207 (1994).

[19] M.-O. Mewes, M. R. Andrews, N. J. van Druten, D. M. Kurn, D. S. Durfee, C. G. Townsend and W. Ketterle, Phys. Rev. Lett. 77, 988 (1996).

[20] L.D. Landau, Phys. Zeitschrift 2, 46 (1932).

[21] C. Zener, Proc. R. Soc. Lond. Ser. A 137, 696 (1932).

[22] K.-P. Marzlin and J. Audretsch, Phys. Rev. A. 53, 4352 (1996).

[23] P.L. DeVries, AIP conference proceedings 160, 269 (1986).

[24] The animation can be downloaded from the URL http://www.physics.mq.edu.au/ pmarzlin/index.htm 


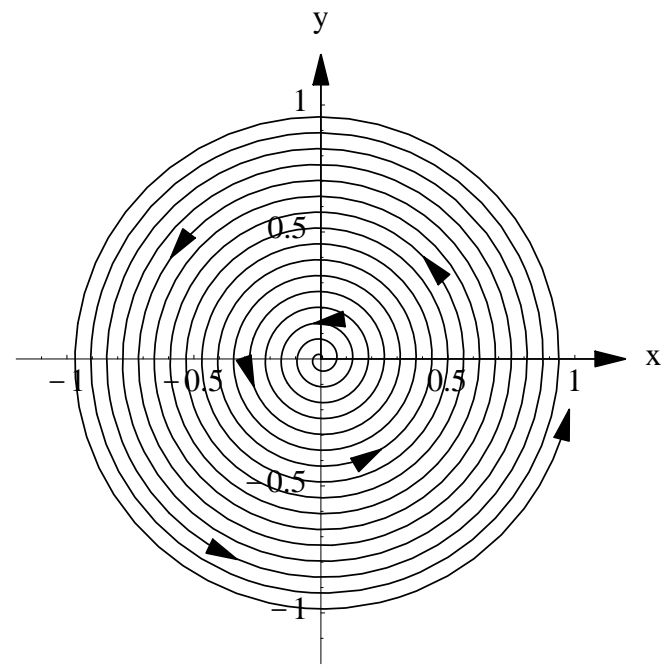

FIG. 1. The trajectory of the center-of-mass of a harmonically trapped condensate under the influence of a rotating homogeneous force. $\mathrm{x}$ and $\mathrm{y}$ are given in units of the trap size $R_{\perp}$. During the rotation the shape of the condensate is preserved.

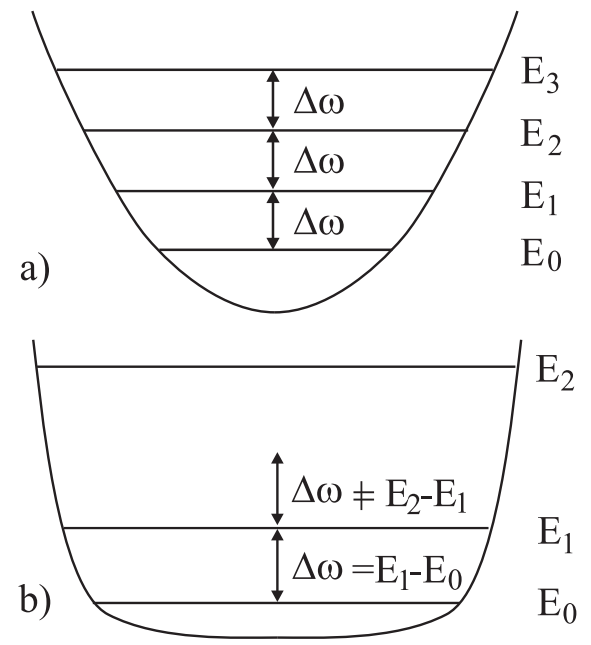

FIG. 2. This diagram shows the ladder of transitions between different trap energy levels due to the optical potential: a) For a harmonic trap all transitions are resonant. b) In an anharmonic trap only the first transition is resonant so that only the first excited vortex state is populated. 


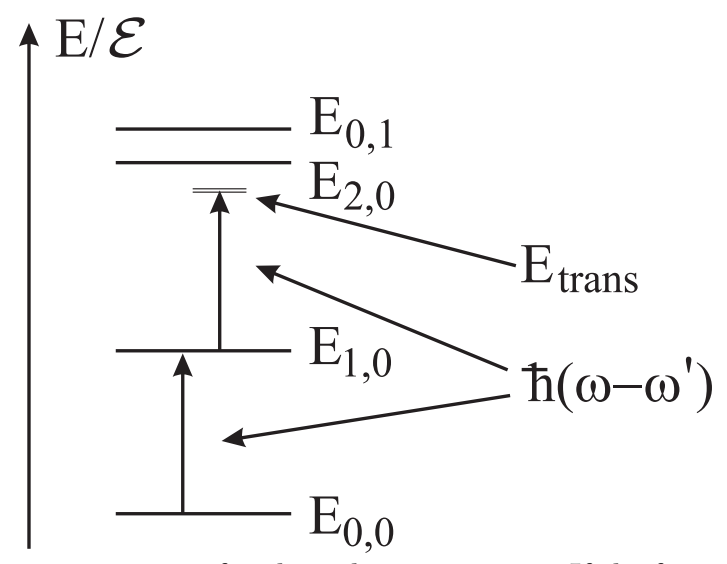

FIG. 3. The lowest lying eigenstates for the anharmonic trap. If the frequency difference $\omega-\omega^{\prime}$ is tuned into resonance with the lowest transition and the optical potential (13) (represented by $E_{\text {trans }}$ ) is weak transitions to higher states are highly suppressed.

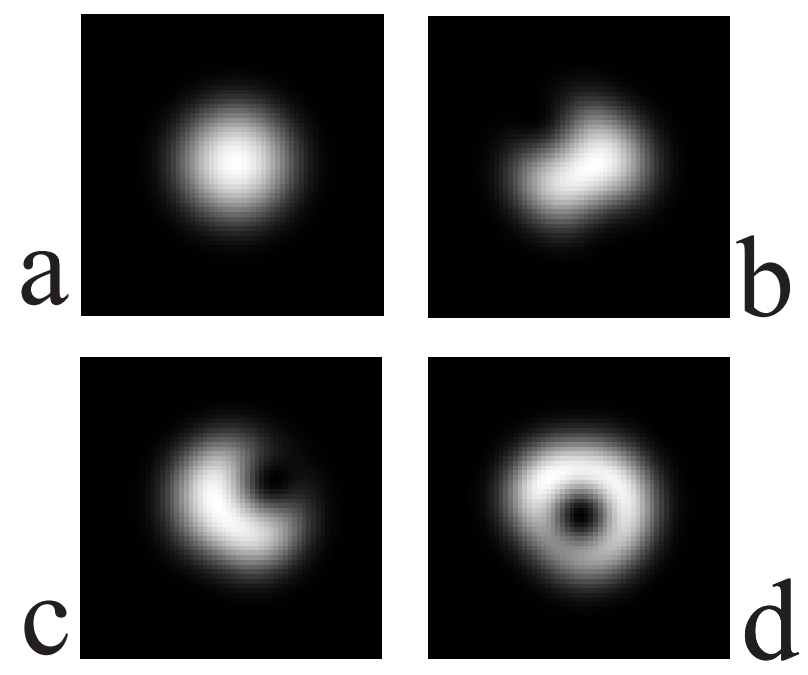

FIG. 4. Numerical simulation of the condensate's time evolution during the vortex creation. The modulus squared of the collective wavefunction is shown at different times (a: $0 \mathrm{~ms}, \mathrm{~b}: 87 \mathrm{~ms}, \mathrm{c}: 142 \mathrm{~ms}, \mathrm{~d}: 247 \mathrm{~ms}$ ). 

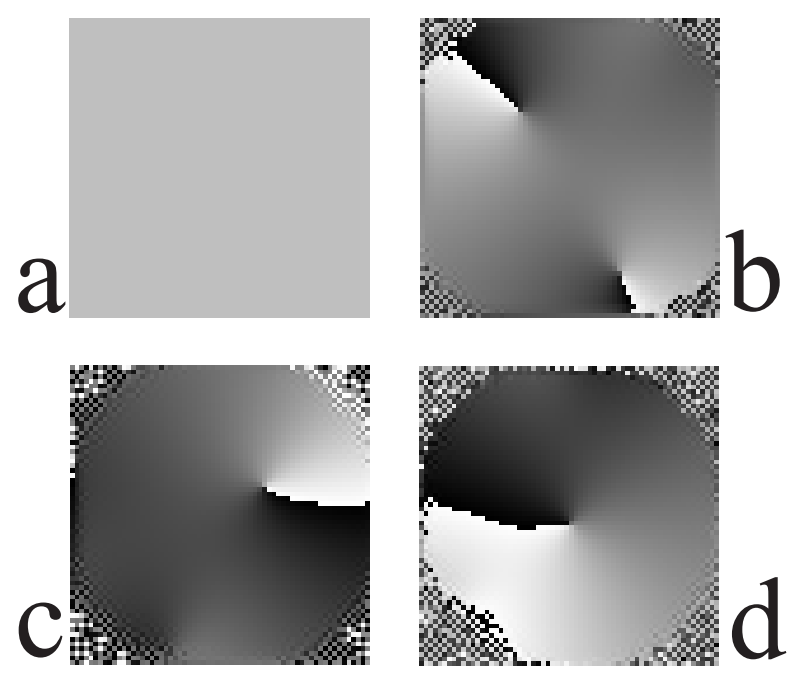

FIG. 5. The same as Fig. 4. The phase of the wavefunction is shown, varying between $\pi$ (white) and $-\pi$ (black). A vortex line corresponds to the end of a sharp borderline between white and black. 